\title{
Graves' disease associated with HIV disease and late immune reconstitution inflammatory syndrome following the initiation of antiretroviral therapy
}

\author{
S Ludgate1, S P Connolly2, D Fennell1, M F Muhamad1', I Welaratne3, A Cotter2,4 and \\ S E McQuaid1,4
}

${ }^{1}$ Department of Diabetes and Endocrinology, 2Department of Infectious Diseases, ${ }^{3}$ Department of Radiology, Mater Misericordiae University Hospital, Dublin, Ireland, and ${ }^{4}$ School of Medicine, University College Dublin, Dublin, Ireland

Correspondence should be addressed to S E McQuaid Email

smcquaid@mater.ie

\section{Summary}

Both human immunodeficiency virus (HIV) and antiretroviral therapy (ART) are associated with endocrine dysfunction (1). The term 'immune reconstitution inflammatory syndrome' (IRIS) describes an array of inflammatory conditions that occur during the return of cell-mediated immunity following ART. Graves' disease (GD) occurs rarely as an IRIS following ART. In this study, we describe the case of a 40-year-old Brazilian female who was diagnosed with HIV following admission with cryptococcal meningitis and salmonellosis. At this time, she was also diagnosed with adrenal insufficiency. Her CD4 count at diagnosis was 17 cells/ $\mu \mathrm{L}$ which rose to 256 cells/ $\mu \mathrm{L}$ over the first 3 months of ART. Her HIV viral load, however, consistently remained detectable. When viral suppression was finally achieved 21 months post diagnosis, an incremental CD4 count of 407 cells/ $\mu \mathrm{L}$ over the following 6 months ensued. Subsequently, she was diagnosed with a late IRIS to cryptococcus 32 months following initial ART treatment, which manifested as non-resolving lymphadenitis and resolved with high-dose steroids. Following the initiation of ART for 45 months, she developed symptomatic Graves' hyperthyroidism. At this time, her CD4 count had risen to 941 cells/ $\mu \mathrm{L}$. She has been rendered euthyroid on carbimazole. This case serves to remind us that GD can occur as an IRIS post ART and typically has a delayed presentation.

\section{Learning points:}

- Endocrinologists should be aware of the endocrine manifestations of HIV disease, in particular, thyroid pathology.

- Endocrinologists should be aware that IRIS can occur following the initiation of ART.

- Thyroid dysfunction can occur post ART of which Graves' disease (GD) is the most common thyroid manifestation.

- GD as a manifestation of ART-induced IRIS can have a delayed presentation.

- Infectious disease physicians should be aware of endocrine manifestations associated with HIV and ART.

\section{Background}

It is estimated that more than 33 million people live with HIV worldwide (1). Thyroid function test abnormalities are common in HIV-infected patients and other associated endocrine disorders include diabetes, adrenal insufficiency, hypogonadism and growth hormone deficiency $(1,2)$. ART is also associated with endocrinopathies, including low bone mineral density, weight gain, insulin resistance, type 2 diabetes mellitus, lipodystrophy and thyroid dysfunction (2). 
IRIS describes an array of inflammatory conditions, indicating the return of cell-mediated immune function. In the setting of HIV infection and initiation of ART, IRIS typically manifests as a paradoxical worsening of underlying opportunistic infection, with common pathogens including Mycobacterium tuberculosis and Cryptococcus neoformans meningitis (3). However, autoimmune thyroid disease can also occur as an IRIS. GD is the most reported manifestation, occurring in approximately $3 \%$ of women diagnosed with HIV and $0.2 \%$ of men (4). In this study, we report a patient with HIV who developed GD as a delayed IRIS presentation.

\section{Case presentation}

A 40-year-old Brazilian lady was diagnosed with HIV in November 2016 after she was admitted to our tertiary centre with a 2-week history of fevers, headache and persistent vomiting. Past medical history included oesophagitis. She was nulliparous with no reported miscarriages and reported a regular menstrual cycle. Blood cultures were positive for Salmonella enteritis and initially grew a yeast that was subsequently identified as $C$. neoformans. Following normal CT brain, lumbar puncture was cryptococcal antigen positive and cerebral spinal fluid (CSF) samples subsequently cultured C. neoformans, confirming a diagnosis of cryptococcal meningitis. She was prescribed 2 weeks of IV ceftriaxone for salmonella bacteraemia, i.v. flyctosine and liposomal amphotericin B followed by fluconazole and high-dose steroids for Cryptococcus meningitis. HIV antibody/antigen was positive; nadir CD4+ and CD8+ T-cell counts were 17 cells/ $\mu \mathrm{L}(4 \%$, reference range: $540-1660)$ and 145 cells/ $\mu \mathrm{L}(7 \%$, reference range: 270-930), respectively; and initial HIV viral load was 1804502 copies/mL.

Four weeks into admission, the patient became persistently hypotensive and developed nausea, vomiting, weakness and worsening fatigue. A diagnosis of primary adrenal insufficiency was made (peak cortisol post Synacthen ${ }^{\circ}$ of $407 \mathrm{nmol} / \mathrm{L}$, ACTH $111 \mathrm{ng} / \mathrm{L}$ (7-63)). Adrenal and TPO antibodies were negative, and CT adrenal showed no infiltrative process (Fig. 1). Symptoms resolved on hydrocortisone replacement.

ART (darunavir/ritonavir plus tenofovir alafenadmide/ emtricitabine) was deferred until 5 weeks post admission. Her CD4+ and CD8+ count increased to 153 cells/ $\mu \mathrm{L}(18 \%)$ and 47 (40\%) following 3 months of ART and remained stable around this level over the following months. HIV viral load remained consistently detectable at low levels for 17 months following the initiation of ART. No resistance-

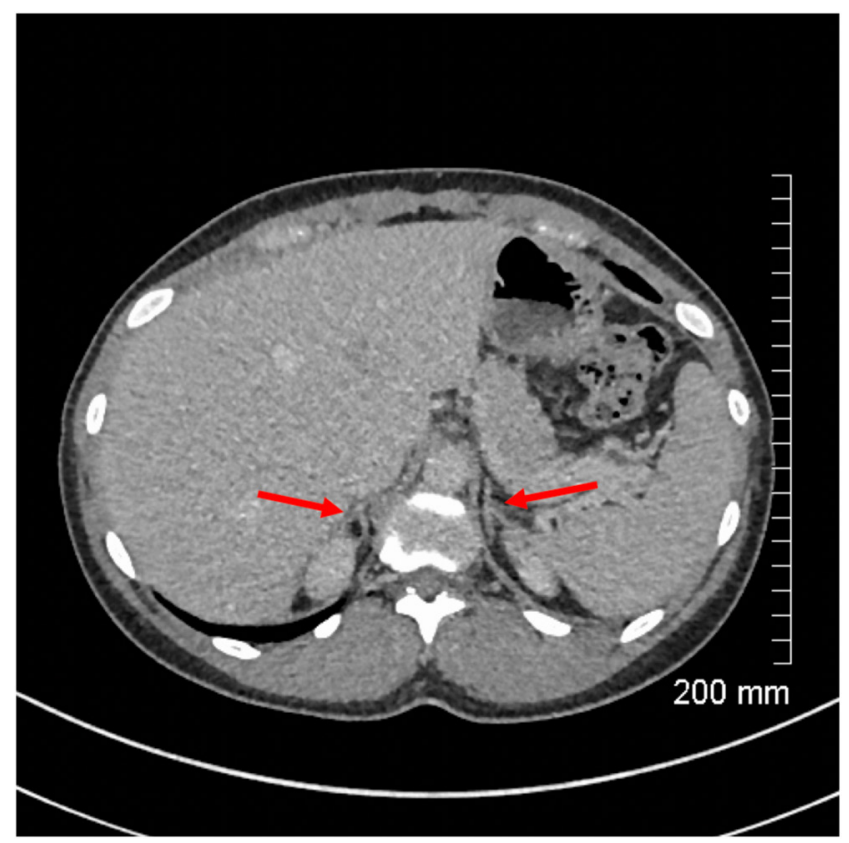

Figure 1

CT abdomen performed in 2016 demonstrating structurally normal adrenal glands (red arrows) at the time of diagnosis with no infiltrative process.

associated mutations were found on HIV-1 genotypic antiretroviral resistance testing.

Nineteen months post original diagnosis, in June 2018, she presented with cervical lymphadenopathy while abroad. She underwent a full investigation including a biopsy without a clear diagnosis. Commencement of high-dose steroids resulted in full resolution of cervical lymphadenopathy. HIV viral load became detectable at 47 copies $/ \mathrm{mL}$, and $\mathrm{CD} 4+$ count remained below the normal range at 219 cells/ $/ \mathrm{L}$. ART was switched to Symtuza $^{\circledast}$ (darunavir/cobicistat/emtricitabine/tenofovir alafenamide) in August 2018, and CD4 count increased from 219 to 626 cells/ $\mu \mathrm{L}$ over 6 months.

In July 2019, 32 months following original diagnosis, she re-presented with a recurrence of cervical lymphadenopathy (CD4+ count 585 cells $/ \mu \mathrm{L}$, undetectable HIV viral load). She underwent extensive workup, including a repeat lymph node biopsy which showed occasional fungal forms highly suspicious for Cryptococcus; however, the culture was negative. Following the investigation and lack of response to prolonged courses of fluconazole, a late immune reconstitution inflammatory syndrome (IRIS) to cryptococcus was diagnosed, with complete resolution of lymphadenopathy on high-dose steroids.

Forty-five months after original ART initiation and 18 months post viral suppression, she developed classical hyperthyroidism symptoms with weight loss, tremor and 
hair thinning with free T4 (FT4) $28.9 \mathrm{pmol} / \mathrm{L}$ (9-20) and thyroid-stimulating hormone (TSH) $<0.01 \mathrm{mIU} / \mathrm{L}(0.35-$ 4.94) (Table 1) (Fig. 2). A diagnosis of GD was confirmed with a positive TSH receptor antibody (TRAB) $3.7 \mathrm{IU} / \mathrm{L}$ $(<1.8)$. Thyroid peroxidase antibodies were negative. She had no extra-thyroidal manifestations. Prior TFTs and thyroid imaging were normal. Family history was negative for thyroid and autoimmune disease. As our patient had no symptoms of COVID-19 infection, there was no requirement to test for PCR, IgG or IgM of SARS-COV2. She was commenced on carbimazole $10 \mathrm{mg}$ daily orally with education on the adverse effects. Thyroid ultrasound showed a structurally normal gland with increased vascularity (Fig. 3A, B, C, D and E).

\section{Outcome and follow-up}

Our patient continues on ART and remains well controlled from an HIV perspective with a normal CD4 count and undetectable viral load under the care of the infectious diseases team.

Her hyperthyroidism has responded well to carbimazole, and the carbimazole dose has been titrated down to $5 \mathrm{mg}$ daily orally based on recent TFTs (FT4 10.5 $\mathrm{pmol} / \mathrm{L}$, TSH $3.11 \mathrm{mIU} / \mathrm{L}$ ) (Table 1). She continues to follow-up in the endocrine outpatient clinic. TRAB will be repeated after 18-24 months of treatment with an attempt to cease carbimazole at this point if possible. Our patient is also regularly followed up for her adrenal insufficiency and is stable on hydrocortisone $10 \mathrm{mg}$ twice daily. She has had no adrenal crisis, and her renin is satisfactory at $1.8 \mathrm{nmol} /$ $\mathrm{L} / \mathrm{h}$ with sodium in the normal range.

\section{Discussion}

Owing to the myriad of clinical presentations of IRIS, a broad differential diagnosis needs to be excluded before

Table 1 Table of thyroid function tests prior to and throughout treatment with ART.

\begin{tabular}{|c|c|c|}
\hline Months post original ART & TSH, mIU/L & FT4, pmol/L \\
\hline-1 & 1.84 & 9.8 \\
\hline 0 & 0.45 & 12.5 \\
\hline 1 & 1.79 & 10 \\
\hline 16 & 1.75 & 10.3 \\
\hline 28 & 0.78 & 10.8 \\
\hline 36 & 0.46 & 10.3 \\
\hline 45 & $<0.01$ & 28.9 \\
\hline 48 & 0.01 & 12 \\
\hline 51 & 3.11 & 10.5 \\
\hline
\end{tabular}

Reference range for TSH: 0.35-4.94 mIU/L; FT4: 9-20 pmol/L.

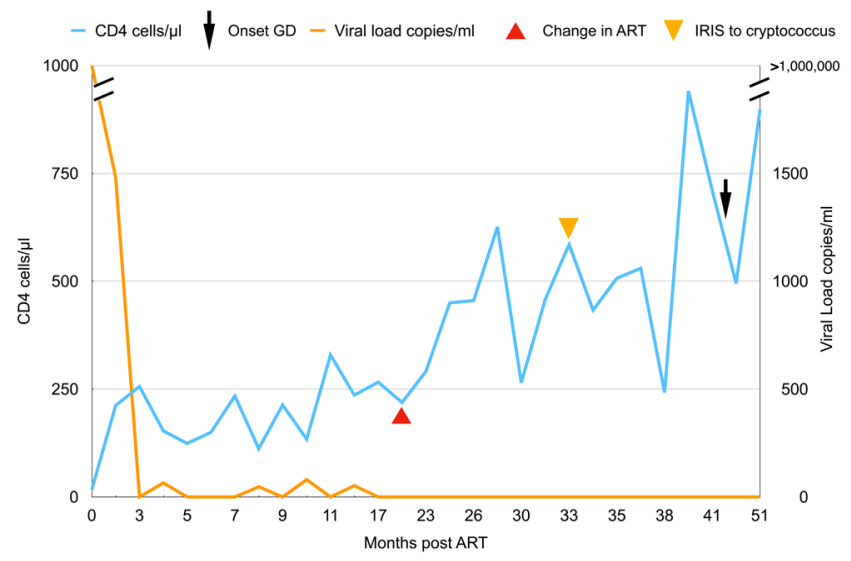

Figure 2

Graph of CD4+ cells $/ \mu \mathrm{L}$ and viral load copies/mL log against months since initiation of ART.

a diagnosis of IRIS can be finalised. French et al. stipulate that IRIS cases must demonstrate initially advanced HIV with a low CD4+ count with positive virologic and immune response to ART and clinical evidence of an inflammatory condition among a number of other criteria (3). The timing of IRIS varies with the inciting pathogen. Our patient developed a delayed IRIS to cryptococcus 32 months following initial ART which is rare; however, it has previously been described in the literature (5).

While abnormal TFTs are relatively common in patients with HIV, the rate of overt hyperthyroidism does not appear to differ from the general population. While some consider that autoimmune disease following ART is a coincidence, GD following ART has been reported in over 70 patients treated for $\operatorname{HIV}(4,6,7,8)$. GD occurs in approximately $3 \%$ of women diagnosed with HIV and $0.2 \%$ of men (4). While this represents a 1.5 - to 2 -fold increase from the general population, GD as an IRIS is more common following alemtuzumab treatment for multiple sclerosis where the incidence of thyroid autoimmunity, including GD, is 30-40\% (9).

While the pathogenesis for GD following ART is not fully understood, a number of possible mechanisms have been described. One mechanism is that CTLA-4 may play a role in T cell tolerance to thyroid autoantigens $(6,10)$. Thymic dysfunction has also been demonstrated (11). French et al found that soluble CD30, a marker of Th2 immune response, occurred alongside a rise in TRAB. The patient with post-ART GD in their study was found to have a rise in CD8+ T cells with naïve immunophenotype (11). The classical onset of GD following ART has been described as 12-36 months following treatment with a median onset of 21 months (2). It has been suggested that CD4+ cells 

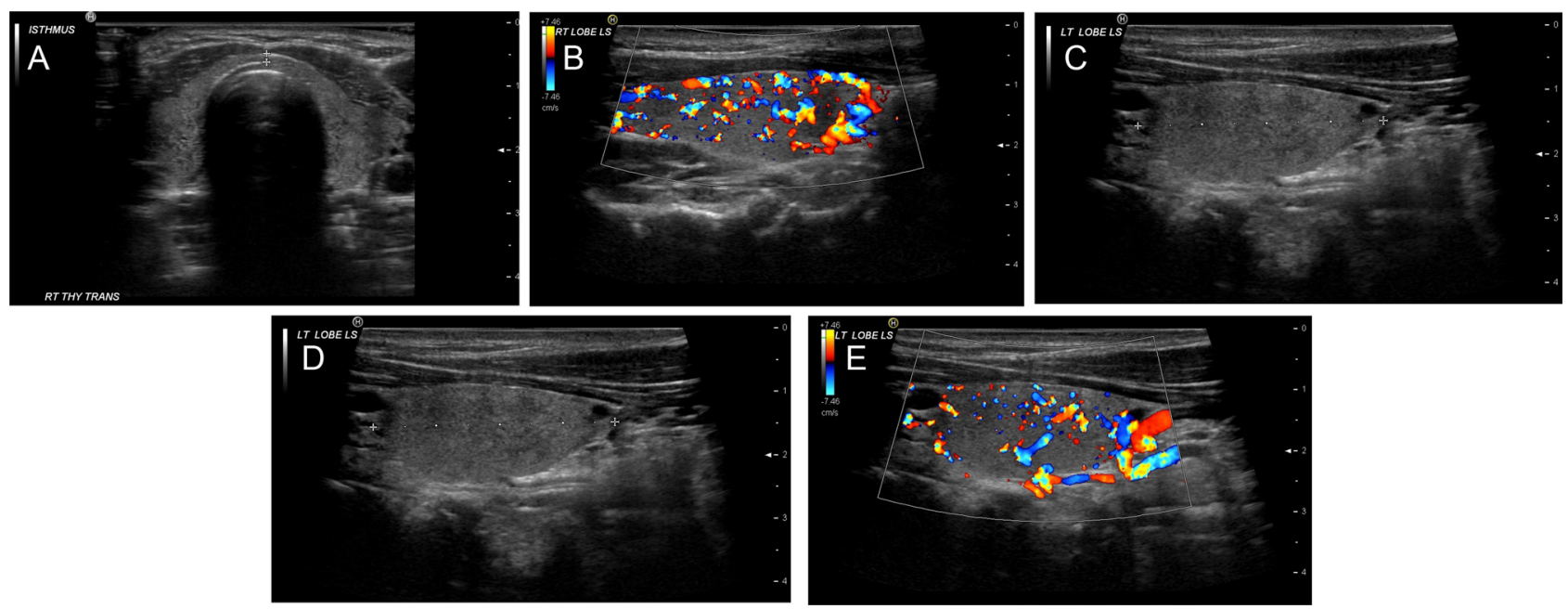

\section{Figure 3}

Ultrasound thyroid. (A) Transverse view of a normal isthmus and both middle lobes. (B) and (C) Longitudinal views of the structurally normal right lobe with increased vascularity on colour Doppler, which can be seen in the setting of Graves' disease. (D) and (E) demonstrating the same findings in the left lobe.

increase in a biphasic pattern following ART initiation (2). A possible explanation for the delayed presentation of GD following ART may be the expansion of naive CD4+ cells occurring months after the initial redistribution of memory CD4 cells from lymphoid tissue (2). Our patient underwent a change in ART 24 months prior to her diagnosis of GD, after which she had a measured increase in CD4+ count of 722 cells $/ \mu \mathrm{L}$ ( 221 cells/ $\mu \mathrm{L}$ at nadir of change in ART to 941 cells/ $\mu \mathrm{L}$ prior to diagnosis).

We cannot be definitive if the GD was of new-onset independent of HIV status or if it occurred as part of an IRIS. However, the diagnosis of documented late IRIS to cryptococcus suggests that the GD is a consequence of ART immune reconstitution. The late-onset occurrence of autoimmunity is a common characteristic of different forms of immune reconstitution syndromes. Two cases of GD 48 months following ART have been reported in the literature $(7,8)$.

Our patient had no extra-thyroidal manifestations. One study has suggested that Graves' orbitopathy can occur in up to $25 \%$ of patients (8).

The management of GD following ART is similar to usual management. To our knowledge, there is no published work on the relapse rates in GD following ART. Our patient also had primary adrenal insufficiency felt to be connected to her cryptococcal infection given that adrenal antibodies were negative. Primary adrenal insufficiency is reported in $17 \%$ of hospitalised HIV inpatients.

This case highlights the importance of remaining vigilant for potential endocrine effects that can occur during clinical follow-up in HIV patients treated with
ART. The occurrence of GD post ART is characterised by a delayed presentation (8). It is important that all physicians be aware of the signs and symptoms of adrenal insufficiency in the setting of non-specific symptoms. Clinicians should also be cognisant of the signs of hyperand hypothyroidism as neither the European AIDS Clinical Society nor the American Academy of HIV medicine currently recommends the routine screening of thyroid function tests either at the initiation of or during ART. In 2019, the European Thyroid Association released guidelines on the management of thyroid dysfunction following immune reconstitution therapy. In these guidelines, it is recommended that TSH should be tested following ART for treatment of HIV; however, routine ongoing testing of TSH/FT4 in such patients is not advised unless clinically indicated by the presence of the symptoms of hypo- or hyperthyroidism (9). Cross-communication between endocrinologists and other specialities remains essential to the co-ordinated care of these patients.

\section{Declaration of interest}

The authors declare that there is no conflict of interest that could be perceived as prejudicing the impartiality of the research reported.

\section{Funding}

This study did not receive any specific grant from any funding agency in the public, commercial or not-for-profit sector.

\section{Patient consent}

Both written and informed consent have been obtained from the patient for publication of this case report. 


\section{Author contribution statement}

$S$ Ludgate - conducted the chart review, prepared the first draft of the manuscript and was involved in the treatment of the patient. S P Connolly - contributing author and infectious disease registrar involved in the treatment of the patient. D Fennell - contributing author to the final draft of the manuscript and endocrinology registrar involved in the treatment of the patient. M F Muhamad - contributing author to the final draft of the manuscript and endocrinology registrar involved in the treatment of the patient. I Welaratne - contributing author and radiology registrar involved in the preparation of the patient's imaging. A Cotter - consultant Infectious Diseases physician and lead infectious disease physician caring for the patient and contributing author to the final draft of the manuscript. S E McQuaid - consultant Endocrinologist caring for patient, review and preparation of the manuscript.

\section{References}

1 Kalra S, Sleim H \& Kotwal N. Human immunodeficiency virus and the endocrine system. Indian Journal of Endocrinology and Metabolism 2011 15 231-233. (https://doi.org/10.4103/2230-8210.85566)

2 Hoffmann CJ \& Brown TT. Thyroid function abnormalities in HIVinfected patients. Clinical Infectious Diseases 200745 488-494. (https:// doi.org/10.1086/519978)

3 French MA, Price P \& Stone SF. Immune restoration disease after antiretroviral therapy. AIDS 200418 1615-1627. (https://doi org/10.1097/01.aids.0000131375.21070.06)

4 Chen F, Day SL, Metcalfe RA, Sethi G, Kapembwa MS, Brook MG, Churchill D, de Ruiter A, Robinson S, Lacey CJ, et al. Characteristics of autoimmune thyroid disease occurring as a late complication of immune reconstitution in patients with advanced human immunodeficiency virus (HIV) disease. Medicine 2005 84 98-106. (https://doi.org/10.1097/01.md.0000159082.45703.90)

5 Hashimoto H, Hatakeyama S \& Yotsuyanagi H. Development of cryptococcal immune reconstitution inflammatory syndrome 41 months after the initiation of antiretroviral therapy in an AIDS patient. AIDS Research and Therapy 2015 12 33. (https://doi. org/10.1186/s12981-015-0075-6)

6 French M \& French MA. Immune reconstitution inflammatory syndrome: a reappraisal. Clinical Infectious Diseases 200948 101-107. (https://doi.org/10.1086/595006)

7 Crum NF, Ganesan A, Johns ST \& Wallace MR. Graves disease: an increasingly recognized immune reconstitution syndrome. AIDS 2006 20 466-469. (https://doi.org/10.1097/01.aids.0000196173.42680.5f)

8 Jariyawattanarat V, Sungkanuparph S \& Sriphrapradang C. Characteristics of Graves disease in HIV-infected patients on antiretroviral therapy. Endocrine Practice 202026 612-618. (https://doi. org/10.4158/EP-2019-0514)

9 Muller I, Moran C, Lecumberri B, Decallonne B, Robertson N, Jones J \& Dayan CM. 2019 European Thyroid Association guidelines on the management of thyroid dysfunction following immune reconstitution therapy. European Thyroid Journal 20198 173-185. (https://doi.org/10.1159/000500881)

10 Kavvoura FK, Akamizu T, Awata T, Ban Y, Chistiakov DA, Frydecka I, Ghaderi A, Gough SC, Hiromatsu Y, Ploski R, et al. Cytotoxic T-lymphocyte associated antigen 4 gene polymorphisms and autoimmune thyroid disease: a meta-analysis. Journal of Clinical Endocrinology and Metabolism 200792 3162-3170. (https://doi. org/10.1210/jc.2007-0147)

11 French MA, Lewin SR, Dykstra C, Krueger R, Price P \& Leedman PJ. Graves' disease during immune reconstitution after highly active antiretroviral therapy for HIV infection: evidence of thymic dysfunction. AIDS Research and Human Retroviruses 200420 157-162. (https://doi.org/10.1089/088922204773004879)

Received in final form 21 July 202

Accepted 14 September 2021 